\title{
Análisis de ciclo de vida de los paneles de lana mineral de vidrio para la construcción de conductos de climatización. Verificación externa
}

\author{
Life cycle assesment for glasswool panels of HVAC ducts. External \\ Verification
}

S. Herranz García $^{(*)}$, J. García Navarro ${ }^{(*)}$

RESUMEN

El presente trabajo expone la situación actual del comportamiento ambiental, a lo largo de su ciclo de vida, de los conductos de climatización realizados con paneles de lana mineral de vidrio. Su uso cada vez es más habitual en la realización de instalaciones de alta eficiencia, sustituyendo frecuentemente a los conductos de chapa metálica revestidos con mantas de lana mineral de vidrio, debido a las ventajas que aportan a la instalación. El análisis del ciclo de vida nos permite cuantificar el impacto medioambiental de estos productos. El procedimiento será realizado utilizando como base la norma UNE-EN 15804:2012+A1, que define el marco común para armonizar la estructura de las Declaraciones Ambientales de Producto en materiales de construcción y da veracidad con base científica a los datos ambientales aportados, al ser verificados por un tercero.

Palabras clave: Conductos, lana mineral de vidrio, climatización, análisis Ciclo de Vida, Declaraciones Ambientales de Producto.

\section{ABSTRACT}

This paper presents the current situation of the environmental behavior, throughout its life cycle, of air conditioning ducts made of glass wool panels. Their use is more and more common in the realization of high efficiency installations, frequently replacing sheet metal ducts coated with glass wool blankets, due to the advantages they bring to the installation. The life cycle analysis allows us to quantify the environmental impact of these products. The procedure will be based on the UNE-EN 15804: 2012 + A1 standard, which defines the common framework for harmonizing the structure of Environmental Product Declarations in building materials and gives veracity scientific based on the environmental data provided, when verified by a third part.

Keywords: Ducts, mineral wool, HVAC, Life Cycle Assessment, Environmental Product Declaration.

(*) Universidad Politécnica de Madrid, (España).

Persona de contacto/Corresponding author: silviaherranz983@hotmail.com (S. Herranz García)

ORCID: http://orcid.org/oooo-0oo3-3819-536o (S. Herranz García); http://orcid.org/oooo-0002-5268-1587

(J. García Navarro)

Cómo citar este artículo/Citation: Herranz García, S., García Navarro, J. (2017). Análisis de ciclo de vida de los paneles de lana mineral de vidrio para la construcción de conductos de climatización. Verificación externa. Informes de la Construcción, 69(548): e232, doi: http://dx.doi.org/10.3989/id.55602.

Copyright: (C) 2017 CSIC. Licencia / License: Salvo indicación contraria, todos los contenidos de la edición electrónica de Informes de la Construcción se distribuyen bajo una licencia de uso y distribución Creative Commons Attribution License (CC BY) Spain 3.o. 


\section{INTRODUCCIÓN}

Los conductos fabricados in situ mediante paneles de lana mineral de vidrio, forman parte del día a día en la realización de instalaciones de alta eficiencia en climatización, tanto en España como en otros países (Figura 1). Desde su aparición en los años 30, en Estados Unidos (1) y su posterior introducción en Europa, más concretamente en España en los años 70, no han dejado de evolucionar, tanto en su proceso de fabricación como en la mejora de sus propiedades técnicas.

El núcleo de estos paneles es de lana mineral de vidrio, conocida desde la época de los egipcios pero no fue hasta principios de siglo XIX cuando empezó a fabricarse tal y como la conocemos hoy (2). Los avances de la fabricación en la lana mineral de vidrio y la necesidad de mejorar la eficiencia de las instalaciones, hicieron posible la aparición de estos paneles, cuya tecnología y diseño les hace idóneos para utilizarse en redes de conductos para distribución de aire en instalaciones de climatización.

El constante aumento de las exigencias de la normativa en temas de eficiencia energética, comportamiento acústico y seguridad frente al fuego, hace que estos paneles mejoren constantemente sus prestaciones técnicas (Conductividad térmica, absorción acústica, reacción al fuego, etc...), todo ello conforme a la norma UNE-EN 14303:2010+A1:2013. «Productos aislantes térmicos para equipos en edificación e instalaciones industriales. Productos manufacturados de lana mineral (MW)».

Diferentes estudios y análisis de los materiales utilizados para la fabricación de redes de conductos (3) y otras soluciones constructivas aplicadas a la edificación (4), concluyen la importancia del conocimiento de comportamiento físico de los mismos para su correcta aplicación y máximo aprovechamiento.

A día de hoy, la información medioambiental de estos paneles es escasa. Tampoco se dispone de datos específicos del ciclo de vida de las redes de conductos para climatización. Sin embar- go, si existen algunas referencias sobre los impactos del diseño de la red de conductos en el coste de su ciclo de vida (5).

Los paneles están sometidos a numerosos ensayos para verificar parámetros óptimos de confort térmico (conductividad térmica), de confort acústico (absorción acústica), de salubridad (proliferación bacteriana, emisión de partículas), de comportamiento mecánico (de pérdida de carga, presión) y de eficiencia energética (estanqueidad).

El análisis de ciclo vida (ACV) es una herramienta que nos permitirá conocer el comportamiento medioambiental de estos paneles a lo largo de su vida útil. El análisis se basará en las declaraciones ambientales de producto (en adelante, DAP) que reflejarán esta información en los diferentes módulos en los que se estructura el inventario.

La información, mediante el análisis de ciclo de vida es fundamental para introducirla en herramientas (6) que se utilizan para realizar una evaluación global del ciclo de vida de un edificio.

\subsection{Antecedentes}

El resultado de la evaluación de la sostenibilidad de un material es complejo de definir con exactitud pero, como premisas fundamentales, se debe tener en cuenta no solo la evaluación medioambiental del ciclo de vida del material sino también su coste y su evaluación social, como se indica en la Life Cycle Initiative, donde se especifica:

Evaluación de la Sostenibilidad del Producto $($ LCSA $)=$ LCA + LCC + SLCA (7)

Siendo:

- LCA: Análisis de Ciclo de Vida de Aspectos Medioambientales

- LCC: Análisis de Ciclo de Vida de Aspectos Económicos

- SLCA: Análisis de Ciclo de Vida de Aspectos Sociales

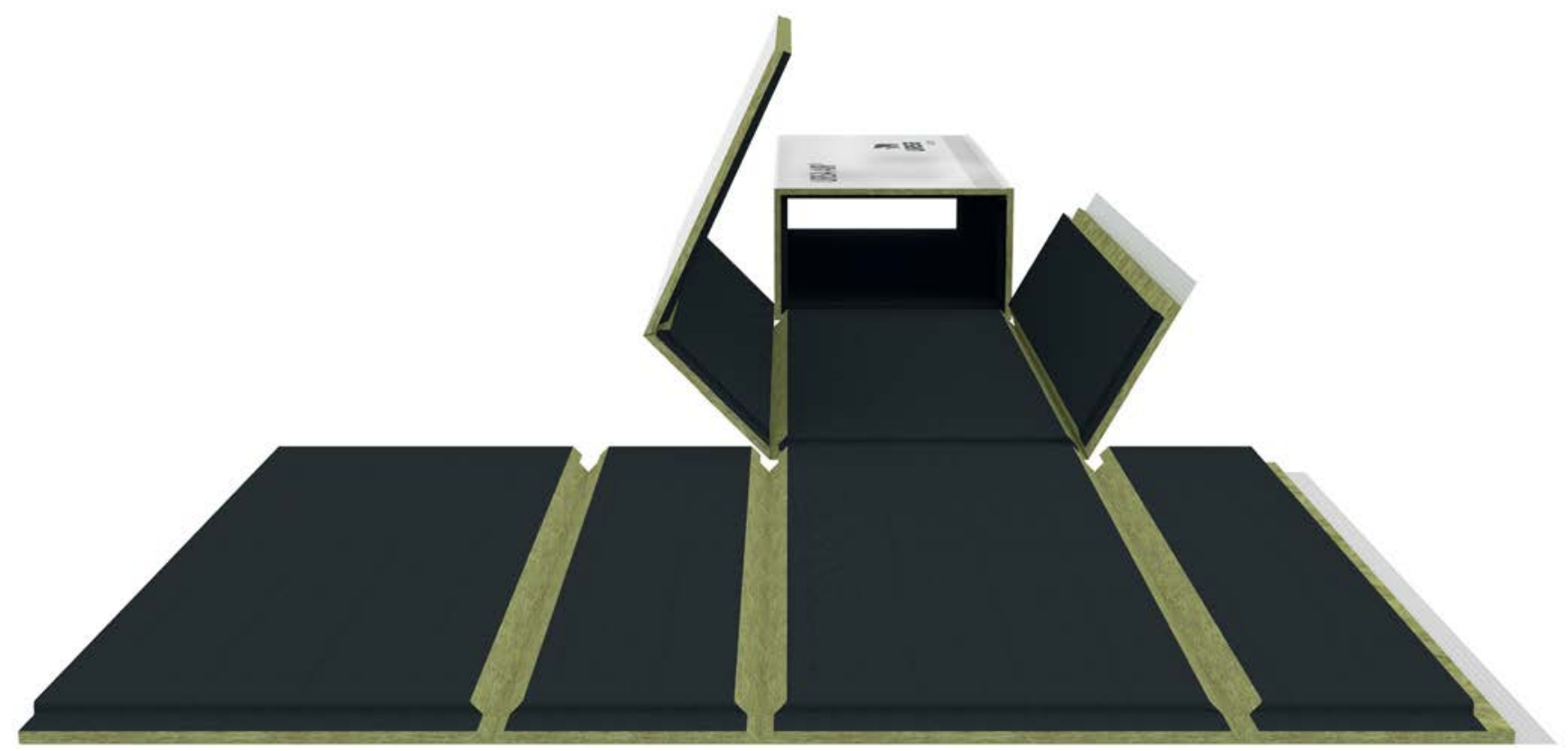

Figura 1. Esquema de construcción de conducto de climatización con panel de lana mineral de vidrio. URSA. 


\subsection{Objetivos y alcance}

El objetivo principal es obtener y analizar la información ambiental de los paneles para la construcción de conductos, cuyo proceso de fabricación difiere al de los de lana mineral utilizados para aislamiento en soluciones constructivas en edificación y de instalación por insuflado. Incluso las normas de obligado cumplimiento a las que son conformes también son diferentes, en el caso de las primeras la norma a la tienen que ser conforme es a la UNE EN 13.162, y en de lana mineral para insuflado es la UN EN 14.064-2 (8).

El alcance de este estudio es definir los parámetros evaluados en el análisis de ciclo de vida de los paneles, reflejados en las declaraciones ambientales y verificadas por un tercero.

Para la verificación de las declaraciones ambientales de producto, se realizó un estudio de los componentes de seis tipos de paneles de la lana mineral de vidrio, a partir del cual se escogieron los cuatro candidatos a obtener los valores máximo y mínimo.

Las premisas (características técnicas de los paneles) que se tomaron en cuenta fueron:

- Aglutinante: Resina entre 11-13 \%

- Densidad: $60-80 \mathrm{~kg} / \mathrm{m} 3$

- Espesores 25 - $40 \mathrm{~mm}$

- Revestimiento exterior:

- Papel kraft / Malla de vidrio / Aluminio reforzado

- Revestimiento interior:

- Velo de vidrio / Malla de vidrio / Aluminio Kraft / Aluminio Reforzado

- Embalaje: en caja de cartón y paletizado a granel

- Paletización: palet estándar de madera 3 x 1,2 m con film de plástico.

- Fábrica: El Pla de Santa María (Tarragona)

Un impacto a tener en cuenta, sería la influencia del factor de ahorro energético en el Análisis de ciclo de vida. Sin embargo esta fuera del alcance de este estudio debido a las diferencias del poder aislante entre las muestras analizadas.

\section{MATERIALES}

El análisis de los elementos que conforman los paneles, se tendrá en cuenta en las primeras etapas de ciclo de vida (producción, transporte y construcción), para su posterior reabsorción en el proceso productivo o formando parte de otros procesos, fomentando así la economía circular. Como herramienta para realizar el estudio se utilizará un software de cálculo de ACV y se tendrá en cuenta toda la normativa actual vigente.

\subsection{Paneles de lana mineral de vidrio para la construcción de conductos de climatización in situ}

En el proceso de fabricación de los paneles se utilizan diferentes materias primas, vidrio reciclado, arena, ligantes, revestimientos... Dependiendo de las características técnicas que se requieran. Además se valorarán los elementos que conforman el embalaje y el paletizado, necesarios para su transporte.

\subsubsection{Características técnicas}

La norma UNE EN 14303 (9) define que las conductividades térmicas (lambdas, $\lambda$ ) se deben ensayar a cuatro temperaturas para proceder a la caracterización de la conductividad térmica 90/90. La resistencia térmica $(\mathrm{R})$ se facilitará a $10^{\circ} \mathrm{C}$, en la menor de las temperaturas.

Otros parámetros que se describen para la caracterización de las muestras son las dimensiones, la reacción al fuego y el código de designación (que incluye parámetros de absorción acústica y resistencia a la difusión del vapor de agua).

En la Tabla 1 pueden observarse los datos técnicos de las diferentes muestras.

\subsubsection{Composición de paneles}

Todos los paneles analizados se componen de:

- Un núcleo de lana mineral de vidrio de alta densidad (6o $80 \mathrm{Kg} / \mathrm{m} 3$ )

- Un revestimiento en la cara interior.

- Un revestimiento en la cara exterior.

Dependiendo del tipo de panel, variarán tanto el espesor del núcleo de lana mineral de vidrio como los revestimientos. Estos, con sus respectivas configuraciones y gramajes, son los que dotan al panel de unas características técnicas u otras.

En la Tabla 2 se detallan los datos técnicos de los diferentes componentes, según el muestreo de los cuatro paneles analizados.

Tabla 1. Características técnicas de las muestras tomadas.

\begin{tabular}{|c|c|c|c|c|c|c|c|}
\hline \multicolumn{2}{|l|}{ Descripción } & \multirow{2}{*}{ Norma } & \multirow{2}{*}{$\frac{\text { Unidad }}{\mathrm{m}}$} & \multirow{2}{*}{$\begin{array}{c}\text { Muestra } 1 \\
3\end{array}$} & \multirow{2}{*}{$\begin{array}{c}\text { Muestra } 2 \\
3\end{array}$} & \multirow{2}{*}{$\begin{array}{c}\text { Muestra 3 } \\
3\end{array}$} & \multirow{2}{*}{$\begin{array}{c}\text { Muestra } 4 \\
3\end{array}$} \\
\hline Largo & & & & & & & \\
\hline Ancho & & & $\mathrm{m}$ & 1,2 & 1,2 & 1,2 & 1,2 \\
\hline Espesor & & & $\mathrm{mm}$ & 25 & 40 & 25 & 25 \\
\hline \multirow{4}{*}{ Conductividad térmica $(\lambda$ 90/90) } & $\mathrm{a} 10^{\circ} \mathrm{C}$ & \multirow{4}{*}{$\begin{array}{l}\text { EN } 12667 \\
\text { EN } 12939\end{array}$} & $\mathrm{~W} / \mathrm{mK}$ & 0,032 & 0,032 & 0,032 & 0,032 \\
\hline & a $24^{\circ} \mathrm{C}$ & & $\mathrm{W} / \mathrm{mK}$ & 0,034 & 0,034 & 0,034 & 0,034 \\
\hline & a $40^{\circ} \mathrm{C}$ & & $\mathrm{W} / \mathrm{mK}$ & 0,036 & 0,036 & 0,036 & 0,036 \\
\hline & a $60^{\circ} \mathrm{C}$ & & $\mathrm{W} / \mathrm{mK}$ & 0,038 & 0,038 & 0,038 & 0,038 \\
\hline Resistencia térmica & a $10^{\circ} \mathrm{C}$ & & $\mathrm{m} 2 \cdot \mathrm{K} / \mathrm{W}$ & 0,78 & 1,21 & 0,78 & 0,78 \\
\hline Reacción al fuego (Euroclases) & & EN 13501-1 & & B s1 do & B s1 do & A2 s1 do & A2 s1 do \\
\hline Código de designación & & & & T5-MV1-AWo,6 & T5-MV1-AWo,6 & $\mathrm{T}_{5}-\mathrm{MV1}$ & $\mathrm{T}_{5}-\mathrm{MV} 1$ \\
\hline
\end{tabular}


Tabla 2. Datos de los componentes de las muestras tomadas.

\begin{tabular}{|c|c|c|c|c|c|}
\hline Descripción & Unidad & Muestra 1 & Muestra 2 & Muestra 3 & Muestra 4 \\
\hline Densidad & $\mathrm{kg} / \mathrm{m}_{3}$ & 75 & 65 & 75 & 75 \\
\hline Naturaleza del ligante & & $\begin{array}{l}\text { Baja emisiones } \\
\text { fenólicas }\end{array}$ & $\begin{array}{l}\text { Baja emisiones } \\
\text { fenólicas }\end{array}$ & $\begin{array}{l}\text { Baja emisiones } \\
\text { fenólicas }\end{array}$ & $\begin{array}{l}\text { Baja emisiones } \\
\text { fenólicas }\end{array}$ \\
\hline \% de Ligante & $\%$ & 12 & 12 & 12 & 12 \\
\hline Tipo de Revestimiento exterior & & Alu Kraft renf+VV & Alu Kraft renf+VV & $\begin{array}{l}\text { Tejido Alu-Vidrio } \\
+\mathrm{VV}\end{array}$ & Alu Kraft renf + VV \\
\hline Gramaje del revestimiento exterior & $\mathrm{g} / \mathrm{m} 2$ & $131+35$ & $131+35$ & $142+35$ & $131+35$ \\
\hline Tipo de Revestimiento interior & & Tejido de Vidrio & Tejido de Vidrio & $\begin{array}{l}\text { Aluminio Kraft } \\
\text { reforzado }\end{array}$ & Velo de Vidrio \\
\hline Gramaje del revestimiento interior & $\mathrm{g} / \mathrm{m} 2$ & $126+25$ & $126+25$ & 131 & 35 \\
\hline
\end{tabular}

\subsubsection{Embalaje}

Dependiendo de las necesidades de cada aplicación a la que van destinados los paneles se realiza el suministro en un tipo de embalaje u otro. Este puede ser en formato caja, paletizado posteriormente o con los paneles paletizados a granel. En ambos casos el palet configurado se sella con un film de plástico para facilitar el transporte y proteger el producto.

En la Tabla 3 se detalla el tipo de embalaje.

\subsection{Normativa referente a los paneles}

Los paneles de lana mineral empleados para diferentes soluciones constructivas deben ser conformes a la norma UNE EN 13162 (10). Sin embargo, los paneles de lana mineral empleados para la construcción de conductos de climatización in situ, son productos destinados a la realización de instalaciones y, por lo tanto, están afectados por el cumplimiento del Reglamento de instalaciones térmicas (RITE). Además deben ser conformes a la norma UNE EN 14.303, y ensayados conforme a la UNE EN 13.403 (11).

\subsection{Software: TEAM}

El software empleado para la realización de este estudio ha sido TEAM TM 5.2 PWC (12), Software que utiliza la metodología del Análisis de ciclo de vida para evaluar diferentes parámetros medioambientales, según la norma ISO 14040 (13) e ISO 14044 y desarrollado por Ecoliban (Francia).

Es una herramienta que permite introducir una base de datos, a partir de la cual generará un modelo inicial que será imprescindible para calcular los parámetros medioambientales definidos en la metodología del presente estudio. El modelo contiene la información relativa a entradas y salidas así como las especificaciones de cada una de las muestras descritas en los apartados anteriores, a las cuales se les asigna la parte proporcional tanto de las materias primas como de la producción.
Hemos empleado también bases de datos secundarias: Módulos genéricos incluidos en las bases de datos de DEAM intrínseca de TEAM 5.2/PWC, Ecoinvent, así como datos específicos proporcionados por los proveedores.

\section{METODOLOGÍA}

Partiendo de esta base, este apartado se focalizara en la evaluación medioambiental del material en su ciclo de vida completo.

\subsection{Normativa para el análisis de datos}

\subsubsection{Normas UNE relacionadas con el ACV}

El conocimiento de la normativa del ACV, permite su correcta aplicación en el proceso de obtención de resultados. Las siguientes normas son las que componen la base del ACV:

La Norma UNE-EN ISO 14040:2006 describe el marco de referencia para el análisis de ciclo de vida donde interaccionan las tres fases del inventario del ciclo de vida, con su posterior interpretación. Como muestra el diagrama siguiente, esta será la metodología que se siga.

- La Norma UNE-EN ISO 14044:2006. Describe los requisitos y directrices del ACV. (14)

- La Norma UNE-ISO/TR 14062:2007 IN. Describe la Integración de los aspectos ambientales en el diseño y desarrollo de productos. (15)

- La Norma EN ISO 14020:2002. Describe los Principios generales de las etiquetas ecológicas y declaraciones ambientales. (16)

- La Norma UNE-CEN/TR 15941:2011 IN. Describe la metodología para la selección y uso de datos genéricos en las declaraciones ambientales de producto. (8)

3.1.2. Reglas de categoría de producto para productos de la construcción

La norma UNE-EN 15804:2012 (17) es la que especifica los requisitos para realizar el ACV y orienta como se tiene que

Tabla 3. Datos del embalaje de las muestras tomadas.

\begin{tabular}{|l|c|c|c|c|c|}
\hline \multicolumn{1}{|c|}{ Descripción } & Unidad & Muestra 1 & Muestra 2 & Muestra 3 & Muestra 4 \\
\hline Film de Plástico & $\mathrm{kg} / \mathrm{m} 2$ & - & 0,05 & - & - \\
\hline Pack cartón & $\mathrm{kg} / \mathrm{m} 2$ & 0,241 & - & 0,241 & 0,241 \\
\hline Panel/Paquete & & 6 & 39 & 6 & 6 \\
\hline Paquete/Palet & un & 7 & 1 & 7 & 7 \\
\hline Masa superficial & $\mathrm{kg} / \mathrm{m} 2$ & 1,875 & 2,6 & 1,875 & 1,875 \\
\hline
\end{tabular}




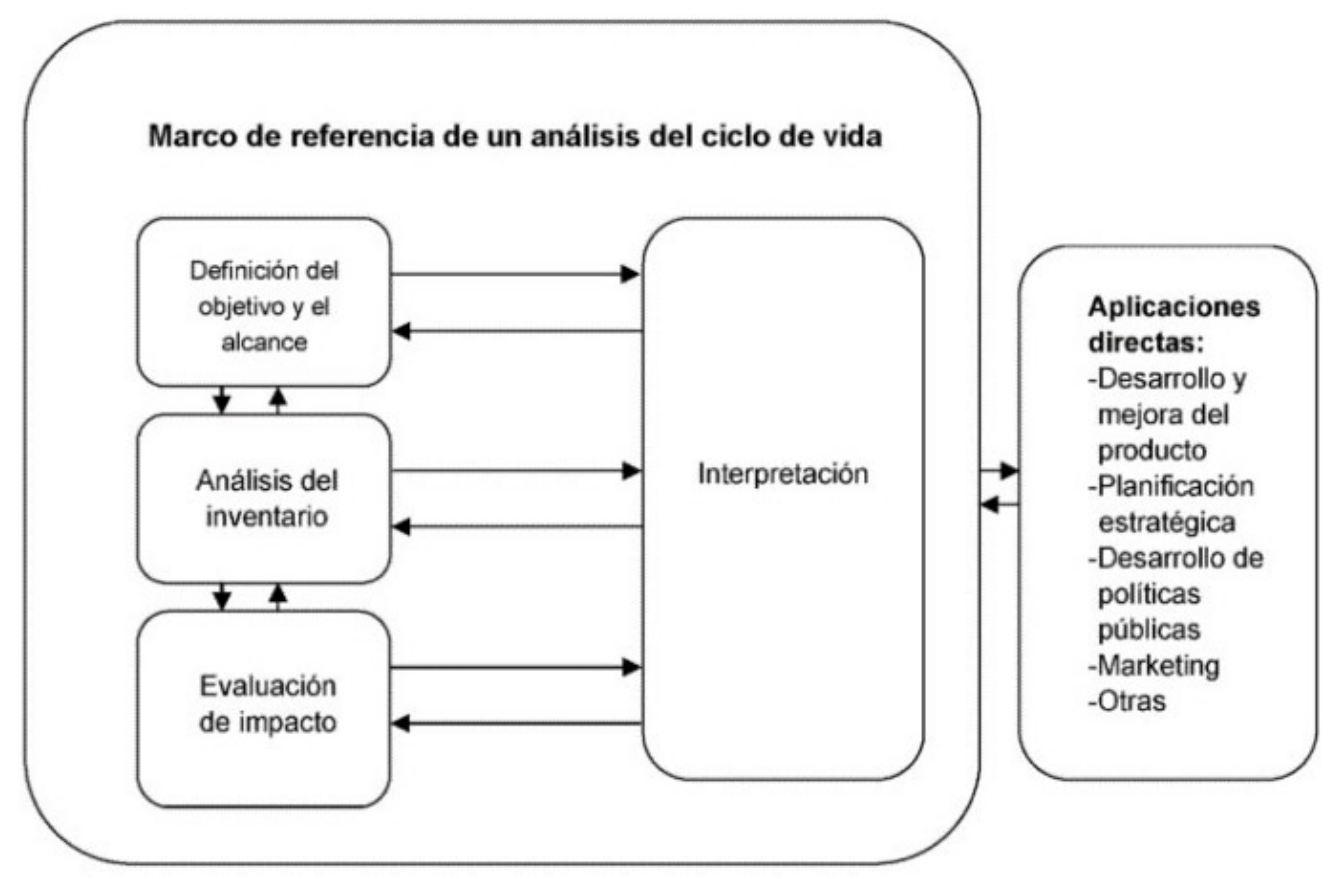

Figura 2. Etapas de un ACV. UNE-EN ISO 14040:2006.

realizar una DAP en productos de construcción (Figura 2). Será la norma base de este estudio.

\subsection{La definición del objetivo y el alcance}

El objetivo de este análisis del ACV es desarrollar las DAP tipo «De la cuna a la tumba» de los paneles de lana mineral de vidrio, para su posterior verificación. En este tipo de DAP es obligatorio tener información en las etapas de productos, del proceso de construcción, de uso y de fin de vida, siendo opcional el módulo D correspondiente al potencial de reutilización, recuperación y reciclaje.

El alcance lo define la unidad funcional, al igual que los parámetros que se van a evaluar.

\subsubsection{Unidad functional}

Se define la unidad funcional de la DAP con el fin de focalizar los elementos que serán de referencia para comenzar el análisis del inventario.

En este estudio, su definición es exactamente:

«Aislamiento térmico de $1 \mathrm{~m}^{2}$ de conducto, asegurando la resistencia térmica $\mathrm{R}=1,21 \mathrm{~m}^{2} \cdot \mathrm{K} / \mathrm{W}$, para una aplicación de construcción de paneles para la construcción de conductos de sistemas de aire acondicionado.» (18) (Pág. 5)

La recopilación de datos, tanto de materias primas como de consumos de energía, es el punto de partida para comenzar con el análisis de inventario, basándonos en el análisis de ciclo de vida del material y teniendo en cuenta las reglas de categoría de producto que se definen en la UNE 15804.

Hay que tener en cuenta:

Asignaciones: Los criterios de asignación se basan en la masa.
Representación geográfica y temporal: País de producción España. País de utilización España. Datos de Producción de 2013.

3.2.2. Impactos medioambientales

Los impactos que se evaluaran serán los siguientes (Tabla 4):

Tabla 4. Impactos medioambientales evaluados en el ACV de los paneles de lana mineral de vidrio.

\begin{tabular}{|l|c|}
\hline \multicolumn{1}{|c|}{ Parámetro evaluado } & $\begin{array}{c}\text { Unidad de } \\
\text { equivalencia }\end{array}$ \\
\hline Potencial de Calentamiento global & $\mathrm{Kg} \mathrm{CO} 2$ equiv. \\
\hline $\begin{array}{l}\text { Potencial de Agotamiento de Ozono } \\
\text { Estratosférico }\end{array}$ & $\mathrm{Kg} \mathrm{CFC11} \mathrm{equiv.}$ \\
\hline Potencial de Acidificación & $\mathrm{Kg} \mathrm{SO}$ equiv. \\
\hline Potencial de Eutrofización & $\mathrm{Kg}$ \\
\hline Potencial de Agotamiento de Recursos Abióticos & $\mathrm{Kg} \mathrm{Sb} \mathrm{equiv.}$ \\
\hline Potencial de Formación de Ozono Fotoquímico & $\mathrm{Kg} \mathrm{etano} \mathrm{equiv.}$ \\
\hline Consumo de energía primaria renovable & $\mathrm{MJ}$ \\
\hline Consumo de energía primaria no renovable & $\mathrm{MJ}$ \\
\hline $\begin{array}{l}\text { Utilización de combustibles secundarios no } \\
\text { renovables }\end{array}$ & $\mathrm{MJ}$ \\
\hline $\begin{array}{l}\text { Utilización de combustibles secundarios } \\
\text { renovables }\end{array}$ & $\mathrm{MJ}$ \\
\hline Consumo de agua dulce & $\mathrm{m} 3$ \\
\hline Producción de residuos & $\mathrm{Kg}$ \\
\hline Peligrosos & $\mathrm{Kg}$ \\
\hline No peligroso & $\mathrm{Kg}$ \\
\hline Radioactivos & $\mathrm{Kg}$ \\
\hline Material de salida para & $\mathrm{Kg}$ \\
\hline Reutilización & $\mathrm{Kg}$ \\
\hline Reciclaje & $\mathrm{Kg}$ \\
\hline Valoración Energética & Kg \\
\hline
\end{tabular}

\subsection{Análisis de inventario}

El diagrama de flujo, que se encuentra en la herramienta, describe el proceso que se llevará a cabo para obtener los re- 
sultados en temas fundamentalmente de materia y energía pero también ampliables a ruido, radiaciones, biodiversidad afectada, utilización del suelo, etc.

Otra función, no menos relevante, es la de dar un mayor conocimiento del proceso y focalizar en la recopilación de datos, con el fin de dar más efectividad, al alcance y a los objetivos del estudio.

El diagrama de flujo está compuesto por subsistemas, que a su vez se dividen en procesos unitarios, que contienen la información más precisa del diagrama.

El análisis del inventario cuantifica las entradas (materias primas y recursos) y las salidas (emisiones ambientales) en el diagrama de flujo. (19)

\subsubsection{Análisis del ACV de los paneles de lana mineral de vidrio para construcción de conductos de climatización}

El ciclo de vida se divide en diferentes etapas, recogidas en diferentes módulos, como son:

\section{Etapa de Producción: Módulos A1-A3}

El proceso de fabricación (Figura 3) consiste en primer lugar en introducir las materias primas (arena, vidrio reciclado, feldespatos...) en el horno de fusión, para conseguir realizar la mezcla fundida que irá pasando por los fibradores, produciendo unos filamentos que se entrelazan. Una vez formado el panel, pasa por el horno de curado y posteriormente se colocan los revestimientos interior y exterior simultáneamente. Como última fase de esta etapa, el panel pasa por la bobinadora y por la paletizadora, para su posterior embalaje. (20)

Los módulos referenciados corresponden a:
- A1 - Fabricación/Obtención de Materias Primas

- A2 - Transporte hasta la fábrica

- A3 - Fabricación

\section{Etapa de transporte y construcción: Módulos A4-A5}

- A4 - Transporte a obra

Se tendrá en cuenta como tipo de transporte un camión con carga útil de $24 \mathrm{Tn}$, con un consumo medio de 38 litros de diésel cada los $100 \mathrm{Km}$. Considerando un $100 \%$ de la capacidad de utilización y un $30 \%$ de retornos vacíos. La capacidad de carga del camión varía en función de la muestra analizada, tomando como consideración un intervalo de entre 104,40 y 165,60 m2/palet con 8 palet por camión.

La información modular, permite adaptar la información de transporte dependiendo de las distancias requeridas para la DAP. Para este estudio se estima una distancia media de 460 $\mathrm{Km}$, de tal manera que compense las distancias más cercanas y lejanas de la fábrica al punto donde se instale.

- A5 - Instalación en Obra

En este módulo se consideran no solo los residuos generados durante la instalación en obra (mermas), sino también las mermas derivadas del despiece antes de la colocación y los residuos de los embalajes, siendo estimados en un $15 \%$.

\section{Etapa de Uso: Módulos B1-B7}

Los paneles no presentan ningún impacto, debido a que una vez instalado no necesita ninguna manipulación.

Estos módulos corresponderían a:

- B1 - Uso o aplicación del producto instalado

- B2 - Mantenimiento

\section{Recepción de materia prima}

Arena (6xido de silice) Feldespato Dolomita Carbonatos Boro Vidrio reciclaje Vidrio reciclaje del proceso interno

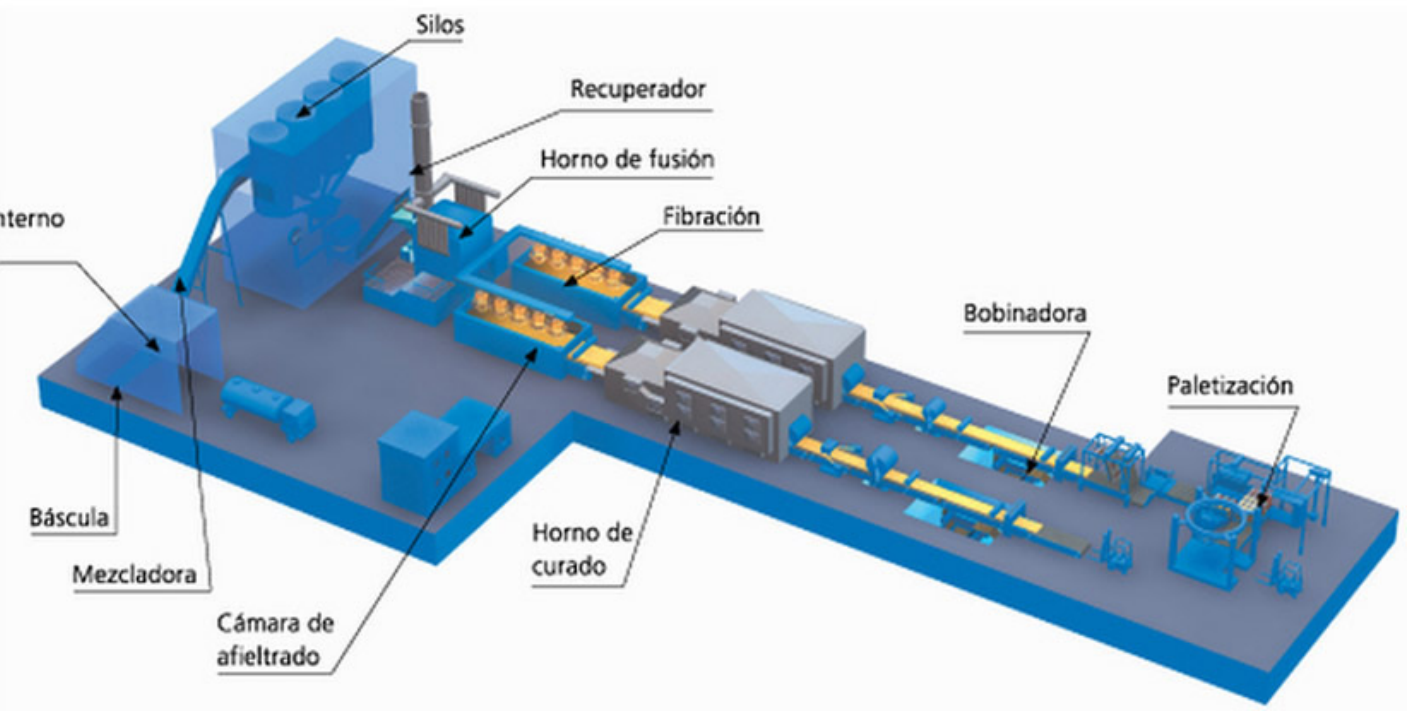

Figura 3. Proceso de Fabricación de los paneles de lana mineral para la construcción de conductos de climatización. URSA. 
- B3 - Reparaciones

- B4 - Reposiciones

- B5 - Rehabilitación

- B6 - Uso operacional de energía

- B7 - Uso operacional de agua

Etapa de fin de vida: Módulos C1-C4

- C1 - Deconstrucción, demolición

Los impactos que producen los paneles son despreciables considerados frente al conjunto de la deconstrucción o demolición del edificio en su totalidad. Se estima que la vida útil de la instalación será igual que la del edificio.

- C2 - Transporte hasta el tratamiento de residuos

La cantidad y procedimiento de deconstrucción será de los $\mathrm{Kg}$ de lana mineral de vidrio de cada producto (mezclados con el resto de materiales de la demolición), considerados como vertidos en vertederos para residuos inertes no peligrosos. Se considera el transporte realizado con un camión de carga útil de $24 \mathrm{Tn}$, con un consumo medio de 38 litros de diésel a los $100 \mathrm{Km}$.

- $\mathrm{C}_{3}$ - Tratamiento de los residuos

Se pueden considerar diferentes escenarios: enterrado en vertedero, que sea recuperado o que sea reciclado. En este caso se ha considerado el caso más desfavorable que será el de eliminado en vertedero.

- C4- Eliminación

El panel está considerado como un residuo no peligroso.

\section{Etapa: Módulo D}

En este módulo se describen escenarios e información técnica adicional. En este caso los beneficios del reciclado de embalajes en A5 no se consideran por su escaso valor e incertidumbre sobre la real revalorización.

\subsubsection{Análisis de etapas}

En el siguiente esquema (Figura 4) se pueden identificar las diferentes etapas y los módulos en los que se divide, al igual que los datos que son obligatorios y/o opcionales, dependiendo del tipo de DAP.

En este estudio se opta por una DAP de la cuna a la tumba, donde se da información de todo el ciclo de vida, desde A1 a C4, siendo opcional la información del módulo D.

\subsection{Evaluación de impactos}

Una vez definidos los impactos a evaluar en el objetivo y el alcance, descritos en el apartado 3.2.2., se extraen del ACV y se analizan, comprobando que son datos coherentes.

\subsection{Verificación externa}

Una vez obtenidos los resultados de los diferentes impactos se procede a la verificación externa de los mismos.

En este caso se recurre a un evaluador externo, de la base de datos INIES (Les données environnementales et sanitaires de référence pour le bâtiment), donde se alojarán los resultados finales obtenidos.

Dicha verificación externa debe realizarse atendiendo a la norma ISO 14025 (22).

\section{Procedimiento:}

Para la verificación de las declaraciones ambientales de producto, se realizó un estudio de todos los componentes de los seis paneles de la lana mineral de vidrio que componen la muestra, para la realización de conductos. A partir ese estudio se seleccionaron los cuatro candidatos a obtener los valores de máximos y mínimos.

Se tomaron en cuenta diferentes características intrínsecas y comunes a cada uno de los seis paneles integrantes de la muestra y se especificaron los valores límite inferior y supe-

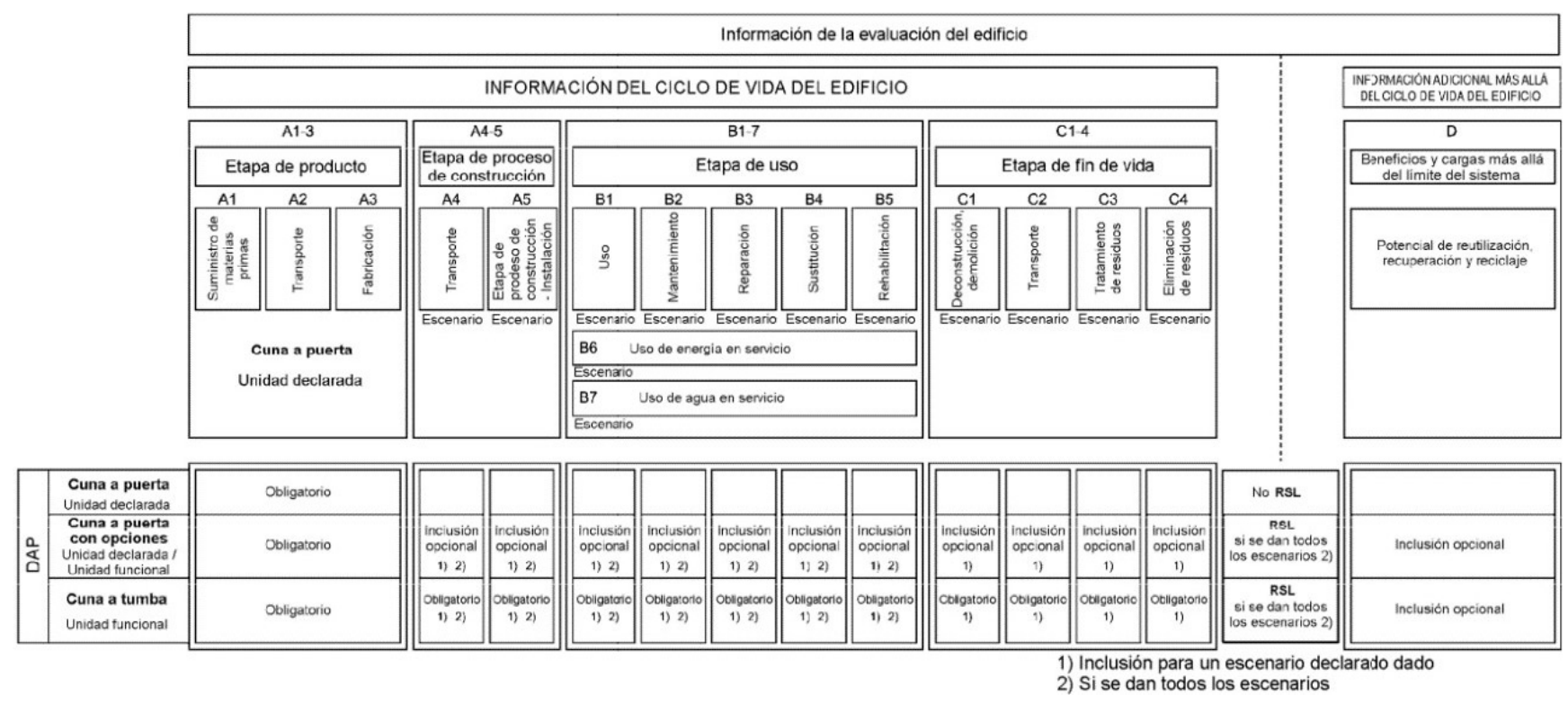

Figura 4. Descripción de las etapas y módulos de un ACV, y tipos de DAP. ISO 21930:2007 (21). 
rior entre los que se encontraban todos ellos englobados. Estas características y sus rangos son:

- Aglutinante: $11-13 \%$

- Densidad: $60-80 \mathrm{~kg} / \mathrm{m} 3$

- Espesores: 25-40 mm

- Revestimiento exterior: Aluminio kraft reforzado ó aluminio Puro

- Revestimiento interior: velo de vidrio y tejido de vidrio ó aluminio

- Embalaje: con y sin caja de cartón

- Paletización: palet estándar de madera $3 \times 1,2$ m con film de plástico.
- Fábrica: El Pla de Santa María (Tarragona)

En base a estos datos, y una vez calculados los parámetros ambientales mediante el software TEAM, se procedió a seleccionar los valores máximos y mínimos de los diferentes impactos, quedando así definido el marco de validación del proceso de verificación.

\section{RESULTADOS}

En la Tabla 5 se muestran los resultados de los diferentes impactos caracterizados con respecto a las cuatro muestras. De estos valores se identifican los valores mínimos

Tabla 5. Resultados de ACV de las muestras, de los mínimos y máximos de cada impacto medioambiental del muestreo.

\begin{tabular}{|c|c|c|c|c|c|c|c|}
\hline & & Muestra 1 & Muestra 2 & Muestra 3 & Muestra 4 & Min & Max \\
\hline Potencial de Calentamiento Global & Kg CO2 equiv. & $5,26 \mathrm{E}+\mathrm{Oo}$ & $7,07 \mathrm{E}+00$ & $5,47 \mathrm{E}+00$ & $4,90 \mathrm{E}+\mathrm{Oo}$ & $4,90 \mathrm{E}+\mathrm{Oo}$ & $7,07 \mathrm{E}+00$ \\
\hline $\begin{array}{l}\text { Potencial de Agotamiento de Ozono } \\
\text { Estratosférico }\end{array}$ & Kg CFC11 equiv & $6,42 \mathrm{E}-\mathrm{O} 7$ & $8,58 \mathrm{E}-07$ & $7,78 \mathrm{E}-07$ & $6,35 \mathrm{E}-07$ & $6,35 \mathrm{E}-07$ & $8,58 \mathrm{E}-\mathrm{O} 7$ \\
\hline $\begin{array}{l}\text { Potencial de Acidificación de suelo y } \\
\text { agua }\end{array}$ & Kg SO2 equiv. & $3, \mathrm{O} 2 \mathrm{E}-\mathrm{O} 2$ & 4,09E-02 & $3,27 \mathrm{E}-\mathrm{O} 2$ & $2,90 \mathrm{E}-\mathrm{O} 2$ & $2,90 \mathrm{E}-\mathrm{O} 2$ & 4,09E-02 \\
\hline Potencial de Eutrofización & $\mathrm{Kg}$ & $5,48 \mathrm{E}-\mathrm{O} 3$ & $7,51 \mathrm{E}-\mathrm{O} 3$ & $5,55 \mathrm{E}-\mathrm{O} 3$ & $5,42 \mathrm{E}-\mathrm{O} 3$ & $5,42 \mathrm{E}-\mathrm{O} 3$ & $7,51 \mathrm{E}-\mathrm{O} 3$ \\
\hline $\begin{array}{l}\text { Potencial de Formación de Ozono } \\
\text { Fotoquímico }\end{array}$ & Kg etano equiv. & $2,44 \mathrm{E}-03$ & $3,29 \mathrm{E}-03$ & $2,57 \mathrm{E}-03$ & $2,24 \mathrm{E}-\mathrm{O} 3$ & $2,24 \mathrm{E}-\mathrm{O} 3$ & $3,29 \mathrm{E}-03$ \\
\hline $\begin{array}{l}\text { Potencial de Agotamiento de Recursos } \\
\text { Abióticos - elementos }\end{array}$ & $\mathrm{Kg}$ Sb equiv. & $1,20 \mathrm{E}-\mathrm{o} 6$ & $1,60 \mathrm{E}-\mathrm{o6}$ & $1,31 \mathrm{E}-06$ & $1,23 \mathrm{E}-06$ & $1,20 \mathrm{E}-\mathrm{O} 6$ & $1,60 \mathrm{O}-\mathrm{O} 6$ \\
\hline $\begin{array}{l}\text { Potencial de Agotamiento de Recursos } \\
\text { Abióticos - combustibles fósiles }\end{array}$ & $\mathrm{Kg}$ Sb equiv. & $8,71 \mathrm{E}+\mathrm{O} 1$ & $1,18 \mathrm{E}+\mathrm{O} 2$ & $8,96 \mathrm{E}+01$ & $7,97 \mathrm{E}+01$ & $7,97 \mathrm{E}+01$ & $1,18 \mathrm{E}+\mathrm{O} 2$ \\
\hline Contaminación del aire & $\mathrm{m}_{3} / \mathrm{UF}$ & $1,01 \mathrm{E}+03$ & $1,36 \mathrm{E}+03$ & $1,06 \mathrm{E}+03$ & $9,70 \mathrm{E}+\mathrm{O} 2$ & $9,70 \mathrm{E}+\mathrm{O} 2$ & $1,36 \mathrm{E}+\mathrm{O} 3$ \\
\hline Contaminación del agua & $\mathrm{m} 3 / \mathrm{UF}$ & $3,13 \mathrm{E}+\mathrm{Oo}$ & $4,05 \mathrm{E}+\mathrm{Oo}$ & $3,17 \mathrm{E}+00$ & $2,75 \mathrm{E}+\mathrm{OO}$ & $2,75 \mathrm{E}+\mathrm{OO}$ & $4,05 \mathrm{E}+\mathrm{Oo}$ \\
\hline $\begin{array}{l}\text { Uso de la energía primaria renovable, } \\
\text { excluyendo los recursos de energía } \\
\text { primaria renovable utilizada como } \\
\text { materia prima }\end{array}$ & MJ & $1,86 \mathrm{E}+\mathrm{Oo}$ & $4,20 \mathrm{E}+\mathrm{Oo}$ & $1,34 \mathrm{E}+\mathrm{Oo}$ & $1,57 \mathrm{E}+\mathrm{OO}$ & $1,34 \mathrm{E}+\mathrm{Oo}$ & $4,20 \mathrm{E}+\mathrm{OO}$ \\
\hline $\begin{array}{l}\text { Uso de los recursos renovables de } \\
\text { energía primaria utilizada como } \\
\text { materia prima }\end{array}$ & MJ & $1,25 \mathrm{E}+\mathrm{O} 1$ & $1,42 \mathrm{E}+\mathrm{O} 1$ & $1,46 \mathrm{E}+01$ & $1,42 \mathrm{E}+\mathrm{O} 1$ & $1,25 \mathrm{E}+\mathrm{O} 1$ & $1,46 \mathrm{E}+01$ \\
\hline $\begin{array}{l}\text { Uso total de los recursos renovables de } \\
\text { energía primaria }\end{array}$ & MJ & $1,43 \mathrm{E}+01$ & $1,84 \mathrm{E}+\mathrm{O} 1$ & $1,59 \mathrm{E}+01$ & $1,58 \mathrm{E}+\mathrm{O} 1$ & $1,43 \mathrm{E}+01$ & $1,84 \mathrm{E}+\mathrm{O} 1$ \\
\hline $\begin{array}{l}\text { Uso de energía primaria no renovable, } \\
\text { excluyendo los recursos energéticos } \\
\text { primarios no renovables utilizados } \\
\text { como materias primas }\end{array}$ & MJ & $7,84 \mathrm{E}+01$ & $1,05 \mathrm{E}+\mathrm{O} 2$ & $8,07 \mathrm{E}+01$ & $7,17 \mathrm{E}+01$ & $7,17 \mathrm{E}+01$ & $1,05 \mathrm{E}+\mathrm{O} 2$ \\
\hline $\begin{array}{l}\text { Uso de fuentes de energía primaria no } \\
\text { renovables utilizados como materias } \\
\text { primas }\end{array}$ & MJ & $1,32 \mathrm{E}+\mathrm{O} 1$ & $1,96 \mathrm{E}+01$ & $1,33 \mathrm{E}+\mathrm{O} 1$ & $1,24 \mathrm{E}+01$ & $1,24 \mathrm{E}+\mathrm{O} 1$ & $1,96 \mathrm{E}+01$ \\
\hline $\begin{array}{l}\text { Uso total de los recursos de energía } \\
\text { primaria no renovable }\end{array}$ & MJ & $9,16 \mathrm{E}+\mathrm{O} 1$ & $1,25 \mathrm{E}+\mathrm{O} 2$ & $9,40 \mathrm{E}+01$ & $8,41 \mathrm{E}+\mathrm{O} 1$ & $8,41 \mathrm{E}+\mathrm{O} 1$ & $1,25 \mathrm{E}+02$ \\
\hline Uso de materiales secundarios & MJ & 9,01E-01 & $1,24 \mathrm{E}+\mathrm{OO}$ & $9,01 \mathrm{E}-\mathrm{O} 1$ & 9,01E-01 & 9,01E-01 & $1,24 \mathrm{E}+\mathrm{Oo}$ \\
\hline $\begin{array}{l}\text { Uso de combustibles secundarios } \\
\text { renovables }\end{array}$ & $\mathrm{MJ}$ & $\mathrm{O}, \mathrm{OOE}+\mathrm{OO}$ & $\mathrm{o}, \mathrm{OoE}+\mathrm{Oo}$ & $\mathrm{o}, \mathrm{OoE}+\mathrm{Oo}$ & $\mathrm{o}, \mathrm{OoE}+\mathrm{Oo}$ & $\mathrm{o}, \mathrm{ooE}+\mathrm{Oo}$ & $\mathrm{O}, \mathrm{OOE}+\mathrm{OO}$ \\
\hline $\begin{array}{l}\text { Uso de combustibles secundarios no } \\
\text { renovables }\end{array}$ & MJ & $\mathrm{o}, \mathrm{ooE}+\mathrm{Oo}$ & $\mathrm{o}, \mathrm{ooE}+\mathrm{Oo}$ & $\mathrm{O}, \mathrm{ooE}+\mathrm{Oo}$ & $\mathrm{o}, \mathrm{ooE}+\mathrm{OO}$ & $\mathrm{O}, \mathrm{OoE}+\mathrm{OO}$ & $\mathrm{O}, \mathrm{OOE}+\mathrm{Oo}$ \\
\hline Uso neto de agua dulce & m3 & $1,57 \mathrm{E}-\mathrm{O} 2$ & $1,93 \mathrm{E}-\mathrm{O} 2$ & $1,48 \mathrm{E}-\mathrm{O} 2$ & 1,6oE-02 & $1,48 \mathrm{E}-\mathrm{O} 2$ & $1,93 \mathrm{E}-\mathrm{O} 2$ \\
\hline Residuos peligrosos eliminados & $\mathrm{Kg}$ & 6,o6E-02 & $7,86 \mathrm{E}-02$ & $5,51 \mathrm{E}-\mathrm{O} 2$ & $4,44 \mathrm{E}-02$ & $4,44 \mathrm{E}-02$ & $7,86 \mathrm{E}-02$ \\
\hline Residuos no peligrosos eliminados & $\mathrm{Kg}$ & $3,65 \mathrm{E}+\mathrm{Oo}$ & $4,96 \mathrm{E}+\mathrm{Oo}$ & $3,68 \mathrm{E}+\mathrm{Oo}$ & $3,52 \mathrm{E}+\mathrm{Oo}$ & $3,52 \mathrm{E}+\mathrm{OO}$ & $4,96 \mathrm{E}+\mathrm{Oo}$ \\
\hline Residuos Radioactivos eliminados & $\mathrm{Kg}$ & 2,08E-04 & $2,81 \mathrm{E}-\mathrm{O} 4$ & $1,97 \mathrm{E}-04$ & $1,77 \mathrm{E}-04$ & $1,77 \mathrm{E}-04$ & $2,81 \mathrm{E}-\mathrm{O} 4$ \\
\hline Componentes para su reutilización & $\mathrm{kg} / \mathrm{UF}$ & $\mathrm{O}, \mathrm{OOE}+\mathrm{OO}$ & $\mathrm{O}, \mathrm{OOE}+\mathrm{OO}$ & $\mathrm{O}, \mathrm{OOE}+\mathrm{OO}$ & $\mathrm{O}, \mathrm{OOE}+\mathrm{OO}$ & $\mathrm{O}, \mathrm{OOE}+\mathrm{OO}$ & $\mathrm{O}, \mathrm{OOE}+\mathrm{OO}$ \\
\hline Materiales para el reciclado & $\mathrm{kg} / \mathrm{UF}$ & $0,00 E+00$ & $\mathrm{O}, \mathrm{OOE}+\mathrm{Oo}$ & $\mathrm{O}, \mathrm{OOE}+\mathrm{OO}$ & $0,00 E+00$ & $\mathrm{O}, \mathrm{OOE}+\mathrm{Oo}$ & $\mathrm{O}, \mathrm{OoE}+\mathrm{OO}$ \\
\hline $\begin{array}{l}\text { Materiales para la recuperación de } \\
\text { energía }\end{array}$ & $\mathrm{kg} / \mathrm{UF}$ & $\mathrm{o}, \mathrm{ooE}+\mathrm{Oo}$ & $\mathrm{O}, \mathrm{ooE}+\mathrm{Oo}$ & $\mathrm{O}, \mathrm{ooE}+\mathrm{Oo}$ & $\mathrm{o}, \mathrm{ooE}+\mathrm{Oo}$ & $\mathrm{o}, \mathrm{ooE}+\mathrm{Oo}$ & $\mathrm{O}, \mathrm{OOE}+\mathrm{OO}$ \\
\hline La energía eléctrica suministrada fuera & $\mathrm{MJ} / \mathrm{UF}$ & $\mathrm{O}, \mathrm{ooE}+\mathrm{Oo}$ & $\mathrm{O}, \mathrm{OoE}+\mathrm{OO}$ & $\mathrm{O}, \mathrm{OoE}+\mathrm{OO}$ & $\mathrm{o}, \mathrm{ooE}+\mathrm{Oo}$ & $\mathrm{O}, \mathrm{OoE}+\mathrm{OO}$ & o,ooE+oo \\
\hline $\begin{array}{l}\text { Energía del vapor suministrado de } \\
\text { fuera }\end{array}$ & $\mathrm{MJ} / \mathrm{UF}$ & $\mathrm{O}, \mathrm{ooE}+\mathrm{Oo}$ & $\mathrm{O}, \mathrm{OoE}+\mathrm{Oo}$ & $\mathrm{O}, \mathrm{OoE}+\mathrm{Oo}$ & $\mathrm{O}, \mathrm{ooE}+\mathrm{Oo}$ & $\mathrm{O}, \mathrm{ooE}+\mathrm{Oo}$ & $\mathrm{O}, \mathrm{OoE}+\mathrm{Oo}$ \\
\hline $\begin{array}{l}\text { Energía del gas del proceso } \\
\text { suministrada fuera }\end{array}$ & $\mathrm{MJ} / \mathrm{UF}$ & $\mathrm{O}, \mathrm{ooE}+\mathrm{Oo}$ & $\mathrm{O}, \mathrm{ooE}+\mathrm{Oo}$ & $\mathrm{O}, \mathrm{OoE}+\mathrm{Oo}$ & $\mathrm{o}, \mathrm{OoE}+\mathrm{Oo}$ & $\mathrm{o}, \mathrm{ooE}+\mathrm{Oo}$ & $\mathrm{o}, \mathrm{OoE}+\mathrm{Oo}$ \\
\hline
\end{tabular}


y máximos, pudiendo éstos no pertenecer a una misma muestra.

Se han identificado para cada uno de los impactos analizados, en color verde los parámetros más optimistas y en rojo los más desfavorables:

Todos los datos facilitados en la Tabla 5 han sido verificados externamente, como exige la normativa, para dar validez a las DAP obtenidas.

\section{DISCUSIÓN}

El análisis de los impactos (Figura 5), se divide en 3 categorías: Impactos ambientales

- Uso de Recursos

- Categorías de Residuos

- Categoría impactos ambientales

En la Tabla 5, se analizan los impactos medioambientales, agrupados por unidades, en relación a las diferentes etapas del ciclo de vida de los paneles, a excepción de la etapa de uso, debido a que los valores son despreciables.

En las cuatro muestras, la etapa predominante es la de producción, excepto en el impacto de residuos eliminados, donde la etapa más predominante es la de fin de vida, siendo las muestra 2, la que refleja mayores valores para estos impactos, seguida las muestras $3,1 \mathrm{y} 4$, respectivamente.

\subsection{Categoría de usos de los recursos}

De la segunda categoría, en las cuatros muestras, el impacto predominante es el del uso total de los recursos de energía primario no renovable, seguido del de consumo de energía primaria no renovable con exclusión de los recursos energéticos primarios no renovables y por último el del uso de fuentes de energía primaria no renovables utilizados como materias primas. Nuevamente es la muestra 2 la que refleja mayores valores para estos impactos, seguida las muestras 3, 1 y 4, respectivamente (Figuras 6 y 7 ).

\subsection{Categoría de Residuos}

De la categoría de Residuos, sólo se obtienen valores en el impacto relativo a residuos no peligrosos eliminados. Nuevamente es la muestra 2 la que genera mayores valores en este impacto, seguida por las muestras $3,1 \mathrm{y} 4$, respectivamente.

\section{CONCLUSIONES}

Más alla de la información del análisis de ciclo de vida, reflejada en los impactos medioambientales de los paneles de lana mineral de vidrio para la construcción de conductos, y de funcionar como un complemento para obtener un valor más preciso en la evaluación ambiental del edificio en su totalidad, debido a la importancia que tienen las instalaciones de climatización, el aporte de este estudio concluye con:

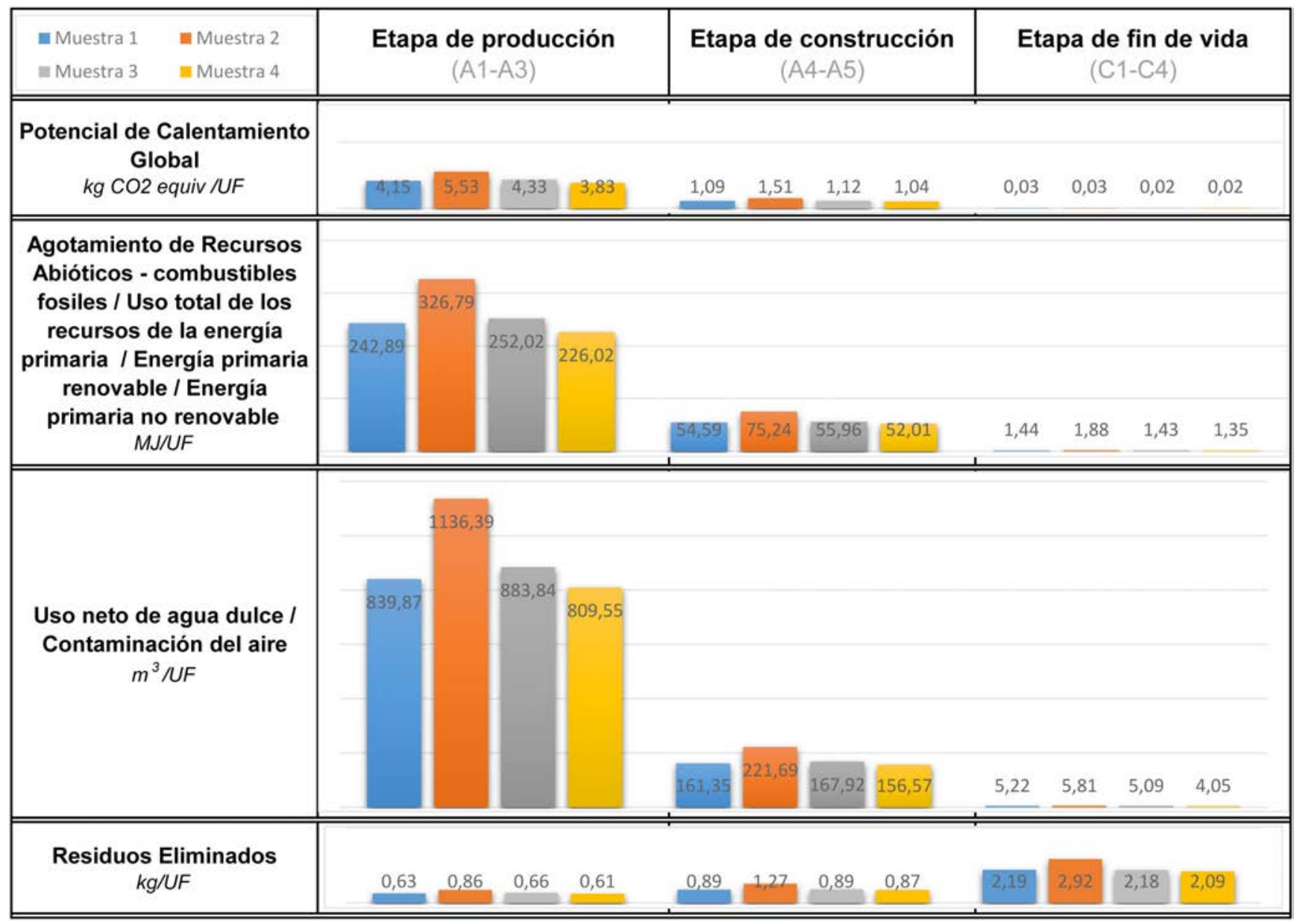

Figura 5. Resultados de ACV según los impactos medioambientales. 


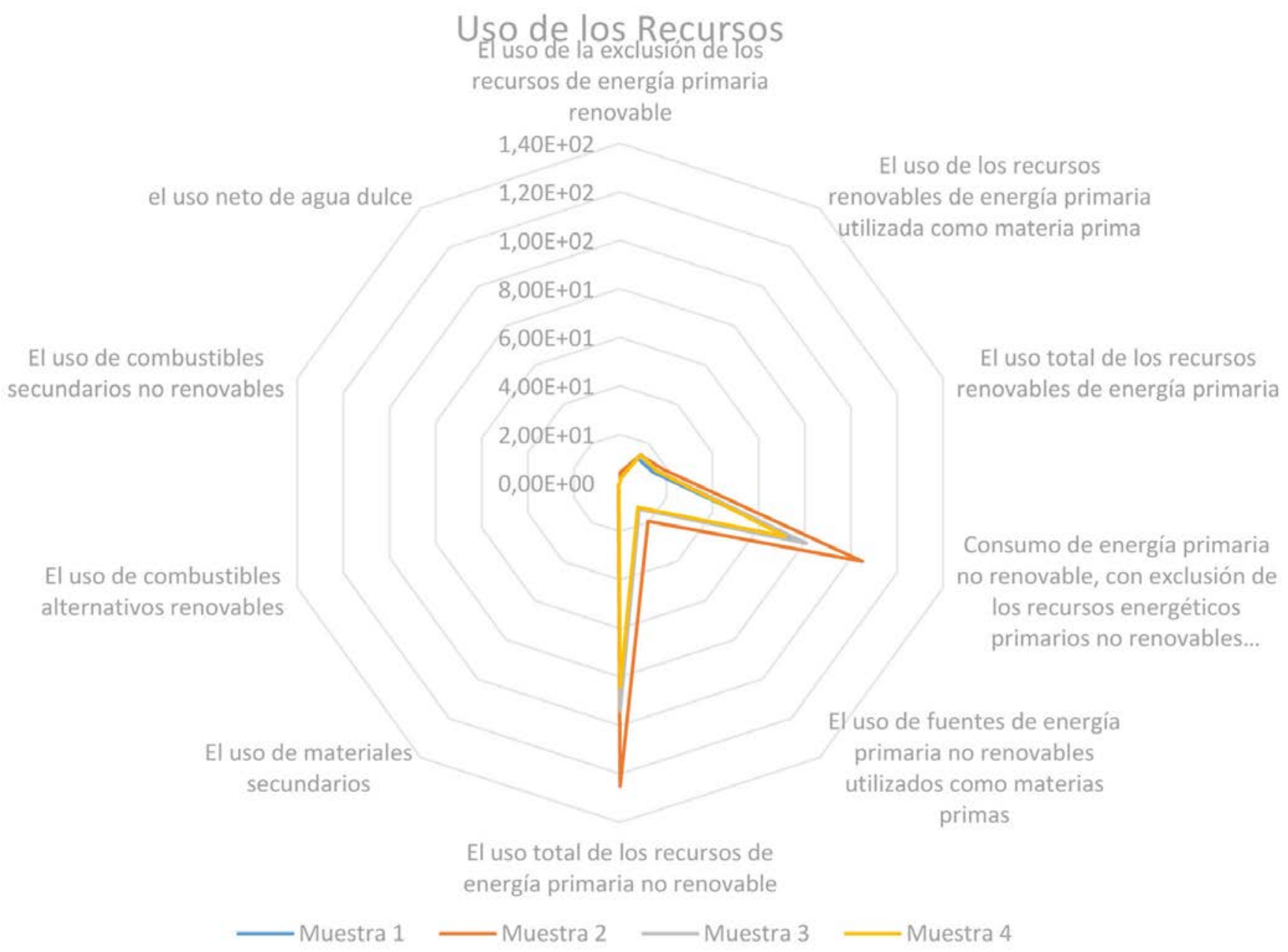

Figura 6. Resultados de ACV según uso de recursos.

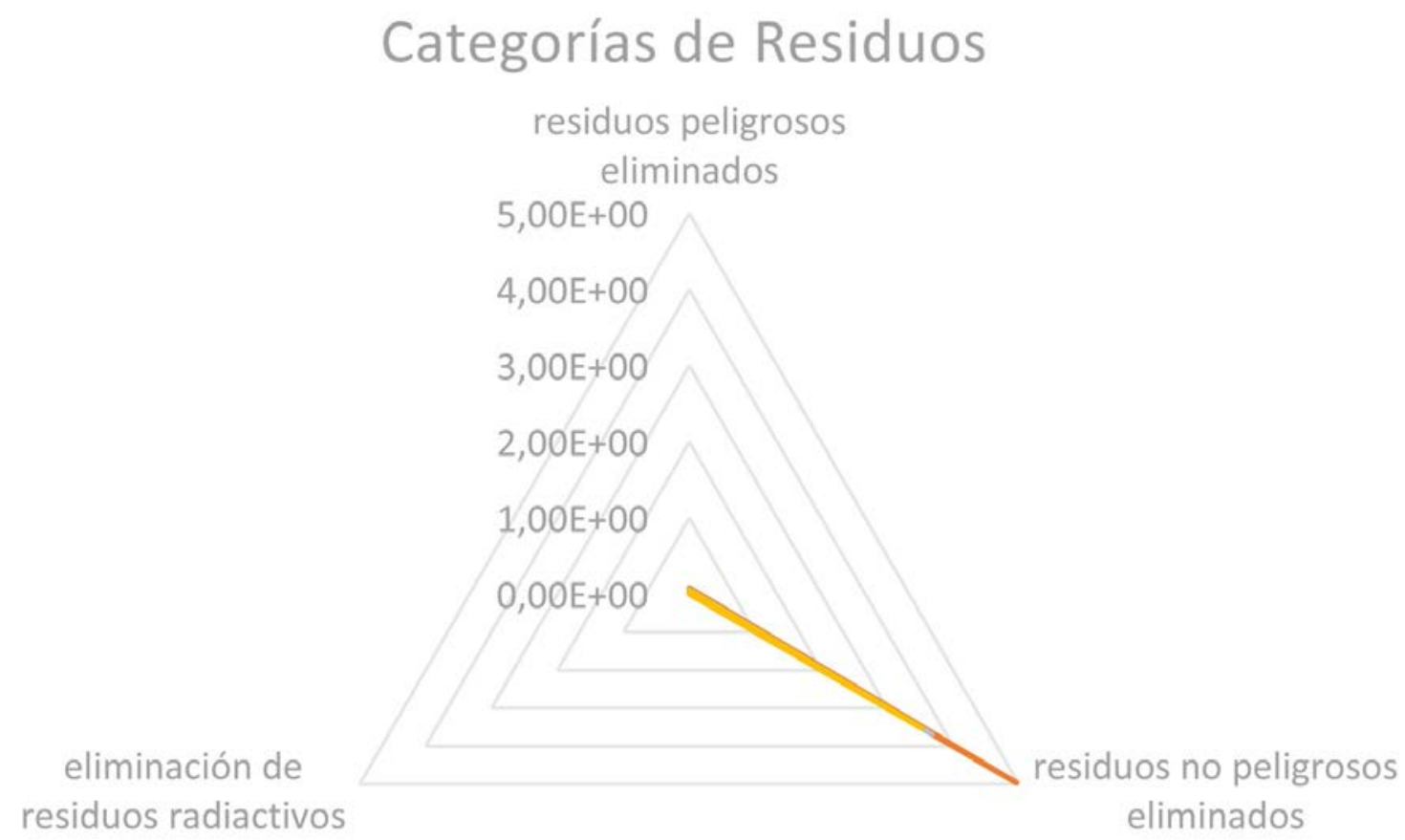

residuos radiactivos

eliminados

Figura 7. Resultados de ACV según categorías de recursos. 
- Los resultados obtenidos nos permiten identificar las etapas e indicadores más críticos durante el ciclo de vida de los paneles de lana mineral de vidrio para la construcción de conductos. Esto nos permite determinar el marco de validación, para la verificación de la declaración ambiental de producto, en cada una de las seis muestras estudiadas.

- Se ha determinado el marco de validación, obteniendo los valores de mínimo y máximo para cada uno de los impactos. El mayor número de valores máximos (más desfavorables medioambientalmente) se ha obtenido en la muestra 2. Por contra, la muestra 4 arroja mayor número de valores mínimos (más favorable medioambientalmente).

- De este análisis se extrae que la etapa más dominante es la de fabricación, excepto en el impacto de residuos eliminados, correspondiente a la etapa de fin de vida del producto. Esta información de las DAP puede convertirse en la base fundamental para el desarrollo de una estrategia en el proceso de producción, que ayude a reducir los impactos medioambientales al máximo, y optimice costes.

- Por otro lado, se deduce que la masa del producto es determinante en el impacto medioambiental del mismo. En este caso, cuando las densidades se mantienen constantes, se traduce en que a mayor espesor mayor impacto.

- Debido a que la masa del producto tiene un gran peso sobre el impacto medioambiental del producto, se observa que el resto de elementos que lo componen, como son los revestimientos interiores y exteriores tienen una incidencia mínima.

\section{AGRADECIMIENTOS}

Este proyecto ha sido realizado dentro del marco de actualización de las DAP de la empresa URSA Ibérica Aislantes S.A.

\section{REFERENCIAS}

(1) North American Insulation Manufacturers Association (NAIMA) [Internet]. Available from: http://insulationinstitute. org/

(2) González Talavera B. Arte y artesanía del vidrio en Segovia. La Real Fábrica de Cristales de la Granja. [Internet]. Fundación EOI, editor. Madrid; 2015. Available from: http://a.eoi.es/artevidriosegovia

(3) Gierzak JB. Duct Liner Materials \& Acoustics. ASHRAE J. 2003;45(12):46-51.

(4) Moretti E, Belloni E, Agosti F. Innovative mineral fiber insulation panels for buildings: Thermal and acoustic characterization. Appl Energy. 2016;169:421-32.

(5) Khoukhi M, Fezzioui N, Draoui B, Salah L. The impact of changes in thermal conductivity of polystyrene insulation material under different operating temperatures on the heat transfer through the building envelope. Appl Therm Eng. 2016;105:669-74.

(6) Isasi XO, Tenorio J, Gazulla C, Zabalza I, Cambra D, Leao S, et al. SOFIAS - Software for life-cycle assessment and environmental rating of buildings. Informes de la Construcción [Internet]. 2016;68(542). Available from: http://dx.doi. org/10.3989/ic.15.055.

(7) UNEP/SETAC Life Cycle Initiative. Global Guidance For Life Cycle Impact Assessment Indicators. Volumen 1. Paris; 2016.

(8) AENOR. UNE-EN 14064-2. Productos aislantes térmicos para aplicaciones en la edificación. Productos aislantes térmicos formados in-situ a partir de lana mineral (MW). Parte 2: Especificación para los productos instalados. Madrid; 2014. p. 24.

(9) AENOR. UNE-EN 14.303:2010+A1 Productos aislantes térmicos para equipos en edifcicación e instalaciones industriales. Productos manufacturados de lana mineral (MW). Especificación. Madrid; 2013. p. 35.

(10) AENOR. UNE-EN 13162:2013+A1. Productos aislantes térmicos para aplicaciones en la edificación. Productos Manufacturados de lana mineral (MW). Especificación. Madrid; 2015. p. 46.

(11) AENOR. UNE-EN 13.403. Ventilación de edificios. Conductos no metálicos. Red de conductos de planchas de material aislante. Madrid; 2003. p. 19.

(12) TEAM TM 5.2 PWC [Internet]. Available from: http://ecobilan.pwc.fr/boite-a-outils/team.html

(13) AENOR. UNE-EN ISO 14040 norma española. Gestión ambiental Análisis del ciclo de vida Principios y marco de referencia. Madrid; 2006. p. 29.

(14) AENOR. UNE-EN ISO 14044 Gestión ambiental. Análisis del ciclo de vida. Requisitos y Directrices. Madrid; 2006. p. 55.

(15) AENOR. UNE-ISO/TR 14062 IN Gestión ambiental. Integración de los aspectos ambientales en el diseño y desarrollo de productos. Madrid; 2007. p. 31.

(16) AENOR. UNE 14020:2000 Etiquetas ecológicas y declaraciones ambientales. Principios generales (ISO 14020:2000). Madrid; 2002.

(17) AENOR. UNE-EN 15804:2012+A1 - Sostenibilidad en la Construcción - Declaraciones Ambientales de Producto - Reglas de Categoría de productos básicas para productos de construcción. Madrid; 2014. p. 65.

(18) URSA. [Internet]. 2016. Available from: www.ursa.es

(19) Fullana, P. Puig R. Análisis de Ciclo de vida. Rubes Edit. Barcelona; 1997.

(20) EURIMA. Mineral Wool I Putting Natural Resources to Work for the Benefit of our Planet [Internet]. Brussels; 2013. Available from: http://www.eurima.org/flipbook/mineralwool/index.html\#/3/zoomed

(21) AENOR. UNE-ISO 21930. Sostenibilidad en la construcción de edifficios. Declaración ambiental de productos de construcción. Madrid; 2010.

(22) AENOR. UNE ISO 14025. Etiquetas y declaraciones ambientales, declaraciones ambientales tipo III, principios y procedimientos. Madrid; 2010. p. 34. 